\title{
Radio Sensing Using 5G Signals: Concepts, State-of-the-art and Challenges
}

\author{
Yunfei Chen, Senior Member, IEEE, Jie Zhang, Senior Member, IEEE, Wei Feng, Senior Member, IEEE, \\ Mohamed-Slim Alouini, Fellow, IEEE
}

\begin{abstract}
Radio sensing has become increasingly important, as the demand for "smartness" is drastically increasing. Unlike conventional sensing, radio sensing uses existing radio signals or devices to passively sense the ambient environment for low cost and wide deployment. In this paper, a comprehensive overview of radio sensing using the recent fifth generation (5G) signals is provided. 5G systems have many merits, such as high frequency, large bandwidth, massive antenna array and dense network, making them ideal for radio sensing. In the overview, basic theories and concepts of 5G radio sensing are first introduced. Then, different state-of-the-art 5G sensing works are discussed based on their applications. These applications show that $5 \mathrm{G}$ radio sensing represents a step change in radio sensing. After that, several open challenges in 5G radio sensing are illustrated with relevant insights. These insights manifest that 5G radio sensing has great potentials to explore.
\end{abstract}

Index Terms-5G, activity recognition, cellular signal, detection, healthcare, localization, radio sensing, safety.

\section{INTRODUCTION}

\section{A. Radio Sensing Concept}

Radio sensing has attracted great research interest recently [1], [2]. Traditional sensing often requires dedicated sensing devices or signals, such as cameras and tags, to perform the sensing tasks [3]. Such methods could be costly, inefficient or inconvenient. For example, cameras require line-of-sight (LOS) so that they perform poorly with obstruction or in case of low visibility. Also, in health monitoring, it is inconvenient to wear tags. These problems can be solved by using radio sensing. A radio receiver is intrinsically a sensor. The radio signals in the ambient environment can be blocked, reflected and diffracted by objects inside it. The presence, the movement or any other changes caused by these objects will lead to variations in the propagation of radio signals. Hence, the received radio signals containing these variations can be used to detect the changes in the ambient environment.

This work is supported in part by EC H2020 DAWN4IoE-Data Aware Wireless Network for Internet-of-Everything under Grant 778305, by the National Key Research and Development Program of China under Grant 2020YFA0711301, and by the National Natural Science Foundation of China under Grant 61941104 and Grant 61922049 . The corresponding author is Wei Feng.

Yunfei Chen is with the School of Engineering, University of Warwick, Coventry, CV4 7AL, UK (e-mail: Yunfei.Chen@warwick.ac.uk).

J. Zhang is with the Department of Electronic and Electrical Engineering, Sheffield University, Sheffield, S1 4DE, UK (e-mail: jie.zhang@sheffield.ac.uk).

Wei Feng is with the Department of Electronic Engineering, Tsinghua University, Beijing, 100084 China (e-mail: fengwei@tsinghua.edu.cn).

Mohamed-Slim Alouini is with the ECE program, King Abdullah University of Science and Technology, Thuwal, Saudi Arabia (e-mail: slim.alouini@kaust.edu.sa).
Radio sensing leverages reflection, diffraction and scattering of radio signals for ubiquitous sensing. Compared with traditional sensing, radio sensing has several advantages. Firstly, its operation is sensor-less, that is, no extra dedicated sensing devices are needed. The radio receiver can work as the sensor. Secondly, it is contactless, and does not require the sensed objects to cooperate. Thirdly, its hardware cost could be low, as one only needs to reuse existing or off-the-shelf radio receivers. Other benefits include privacy protection of object (video or audio could expose personal identity while radio will not) and efficiency (such as working in non-line-of-sight (NLOS) conditions or in the dark). In Issac Asimov's 1983 science fiction book "The Robots of Dawn", he cited "Every time I lift my arm, it distorts a small electromagnetic field that is maintained continuously across the room. Slightly different positions of my hand and fingers produce different distortions and my robots can interpret these distortions as orders" [4]. Radio sensing is making this a reality.

Different radio signals can be used for radio sensing, including FM radio, WiFi, Bluetooth, ZigBee, and cellular. A detailed review of these methods can be found in [2] and [5]. Many of these previous works use WiFi signals due to its low cost and wide availability [6]. However, compared with WiFi signals, cellular signals have several advantages, including wider coverage for both indoor and outdoor applications, longer operational time of base stations, and licensed frequency bands with less interference. These advantages are increasing the popularity of cellular sensing to turn all mobile devices and base stations into distributed sensors for unprecedentedly ubiquitous environment perception [7], [8].

\section{B. $5 G$ for Radio Sensing}

The fifth generation (5G) cellular system adopts a wide range of state-of-the-art technologies, such as massive multiple-input-multiple-output (MIMO), millimeter-wave (mmWave) communications, network densification and deviceto-device (D2D) communications, to enable a variety of use cases [9]. These application scenarios can be mainly summarized into three categories: enhanced mobile broadband (eMBB), ultra-reliable and low latency communications (URLLC), and massive machine type communications (mMTC). For example, eMBB aims to provide high-datarate seamless mobile services for improved user experience, URLLC enables applications requiring stringent latency and reliability, such as autonomous driving and smart manufacturing [10], while mMTC enables Internet of Things (IoT) 


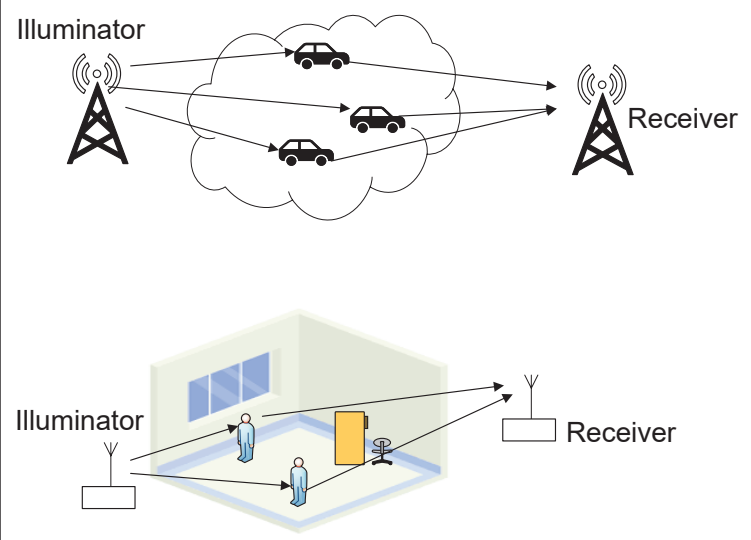

Fig. 1. Diagram of $5 \mathrm{G}$ radio sensing for outdoor and indoor applications.

as another important development in wireless communications [11]. Owing to these attractive applications, $5 \mathrm{G}$ is being rolled out around the world to provide better cellular access. Fig. 1 shows a diagram of $5 \mathrm{G}$ radio sensing for both outdoor and indoor scenarios, where a $5 \mathrm{G}$ base station or small cell can transmit the radio wave that propagates through the environment to be sensed and the radio receiver is used to extract the environment information from the received signals.

The advent of $5 \mathrm{G}$ makes the advantages of using cellular signals for radio sensing even more attractive. $5 \mathrm{G}$ signals have several unique merits that are ideal for reliable and accurate pervasive radio sensing.

1) High Carrier Frequency: 5G uses very high carrier frequency reaching the mmWave bands. The $5 \mathrm{G}$ frequency range one (FR1) includes all existing and new bands below 6 $\mathrm{GHz}$, while the 5G frequency range two (FR2) covers 24.25 $52.6 \mathrm{GHz}$ (albeit quite energy-consuming for a terminal) [12]. The high carrier frequency leads to high ranging resolution to capture more and finer changes in the received signal for better sensing. For example, the velocity resolution in ranging is inversely proportional to the carrier frequency [15]. The high carrier frequency also makes the radio signals more sensitive to absorption loss in the propagation, such as water and chemicals, to allow the sensing of more environmental parameters. Also, high frequency yields smaller antennas to allow for more antennas equipped at the mobile device or base station. The high frequency comes with high penetration loss, reducing multi-path components in the channel to make it sparse and more dependent on the ambient environment, in contrast to previous cellular systems with rich multi-path components. This increases the signal-to-interference ratio of individual multi-path components to make radio sensing easier and better.

2) Large Channel Bandwidth: 5G signals have large channel bandwidth, up to $400 \mathrm{MHz}$ in some cases [13]. This improves radio sensing by increasing its accuracy. For timecritical sensing, large bandwidth gives shorter symbol interval to reduce latency and increase accuracy of time measurements. This is beneficial to time-delay estimation used in some radio sensing applications, as its estimation accuracy is proportional to the bandwidth so that large bandwidth gives very high accuracy. Using time-of-flight (ToF) and time-of-arrival (ToA), the time-delay estimate can be directly transformed to distance estimate. It was shown in [14] that a bandwidth of $100 \mathrm{MHz}$ can achieve a ranging error on the order of centimeters, in contrast to the commonly used global navigation satellite system (GNSS)-based method on the order of meters. The large bandwidth also contributes to finer multi-path resolution to separate closely spaced components from nearby objects.

3) Large Antenna Array: 5G systems use large antenna arrays to implement massive MIMO. For example, Nokia AirScale 64T64R uses 64 antennas. The massive number of antennas or antenna elements provides great spatial flexibility for radio sensing. It enables real-time software-defined reconfiguration of radiation pattern using beam-steering or beamforming. This ability of dynamically changing the radiation pattern allows radio sensing to use different spatial configurations to "view" the same environment or object from different angles, and by comparing or stitching these "views", it can extract the location information of the objects for movement tracking or activity discrimination. The massive number also generates near-pencil beams to increase directivity. The use of large arrays is also well known for directly improving the accuracy of angle-of-arrival (AoA) estimation [16]. The angle resolution is inversely proportional to the number of antennas. It has been reported that, for a bandwidth of $40 \mathrm{MHz}$, it is also possible to achieve a ranging error on the order of centimeters if multiple antennas are used [16].

4) Dense Network: 5G systems have dense deployment of base stations and user equipment, as network densification is a key technology to support large volume of traffic. In an ultra-dense network, a mobile device often has connection to several base stations, at the cost of reduced battery life. This provides spatial diversity for radio sensing, as several reference points will be available. High density also means the availability of a number of neighboring mobile devices to perform collaborative sensing. This is possible for $5 \mathrm{G}$, as it supports D2D communications allowing data exchange between mobile devices directly. High density also gives a high probability of LOS for sensing signals with better qualities.

5) Other Aspects: 5G signals also have other benefits for sensing. For time-non-critical sensing applications, 5G allows the use of cloud radio network for centralized processing of the received signals from different radio heads for spatial processing gain [17]. This is not available in previous cellular systems or in WiFi. For time-critical sensing applications, 5G supports ultra-reliable and low latency communications for fast response, such as collision avoidance. Nevertheless, 5G has limited range and coverage. Also, it has high energy requirement. These might delay the development of $5 \mathrm{G}$ sensing.

Table I gives a list of $5 \mathrm{G}$ characteristics and their advantages and disadvantages for sensing. There has been increasing interest in $5 \mathrm{G}$ radio sensing. For convenience, a list of frequently used abbreviations are summarized in Table II. The main contributions of this work can be summarized as follows:

- A detailed overview of state-of-the-art works on 5G radio sensing is provided with insightful discussion based on their applications.

- It is the first time that different works using $5 \mathrm{G}$ radio 
TABLE I

SUMMARY OF 5G CHARACTERISTICS AND ADVANTAGES

\begin{tabular}{|c|l|c|}
\hline Characteristics & \multicolumn{1}{|c|}{ Advantages } & Disadvantages \\
\hline High frequency & $\begin{array}{l}\text { ligher ranging resolution } \\
\text { for finer sensing, smaller } \\
\text { antenna and higher sensi- } \\
\text { tivity to environment }\end{array}$ & limited range, coverage \\
\hline Large bandwidth & $\begin{array}{l}\text { higher ranging accuracy, } \\
\text { shorter latency, easier sep- } \\
\text { aration of multi-path com- } \\
\text { ponents }\end{array}$ & energy-consuming \\
\hline Dentenna array & $\begin{array}{l}\text { adds degrees of freedom, } \\
\text { increases delay and angle } \\
\text { estimation accuracies }\end{array}$ & high complexity \\
\hline Cloud radio & $\begin{array}{l}\text { increase the number of } \\
\text { reference points and col- } \\
\text { laborators, higher proba- } \\
\text { bility of LOS }\end{array}$ & $\begin{array}{l}\text { centralized processing of } \\
\text { signals from multiple ra- } \\
\text { dio heads for diversity } \\
\text { gain }\end{array}$ \\
\hline Fog computing & $\begin{array}{l}\text { distributed processing to } \\
\text { reduce latency and offload } \\
\text { computing tasks }\end{array}$ & high overhead \\
\hline
\end{tabular}

sensing are compared in terms of methods and results based on their applications. The comparison shows the pros and cons of the relevant works to suggest possible future improvements.

- Open challenges in 5G radio sensing are outlined based on the overview to point out the shortcomings of existing works and therefore, the future research directions.

\section{Structure of the Paper}

This paper provides a comprehensive overview of the growing field on 5G radio sensing. It focuses on sensing using the 5G signals only. State-of-the-art works up to December 2020 will be reviewed. To start with, we will discuss several fields related to $5 \mathrm{G}$ radio sensing to distinguish them and to define the scope of this paper. Then, several basic but important theories and concepts for signal processing in different sensing applications will be introduced. After that, state-ofthe-art works on $5 \mathrm{G}$ radio sensing will be discussed based on their applications, including healthcare, safety and positioning. Finally, open challenges in this relatively new field will be outlined, followed by concluding remarks. Fig. 2 gives the structure of the paper.

\section{Related FieldS AND SCOPE}

Before we proceed to discussing $5 \mathrm{G}$ radio sensing, it is important to distinguish it from several relevant fields that are similar to $5 \mathrm{G}$ radio sensing.

The first field is radio sensing using other wireless signals, such as $\mathrm{WiFi}$, Bluetooth and previous generations of cellular signals. For example, WiFi sensing is a very active research field [18]. Interested readers are referred to [18] and the references therein for WiFi sensing. They are not the focus of this paper. First, there have already been quite a few comprehensive overviews on WiFi sensing, and there is no need to repeat them here. Second, despite some disadvantages, 5G has many unique advantages over WiFi signals, such as
TABLE II

List of Frequently USED AbBREVIATIONS

\begin{tabular}{|c|c|}
\hline $5 \mathrm{G}$ & Fifth generation \\
\hline AoA & Angle-of-arrival \\
\hline AP & Access point \\
\hline CPCL & Cooperative passive coherent location \\
\hline CSI & Channel state information \\
\hline CSQ & Cellular signal quality \\
\hline DKA & Diabetic ketoacidosis \\
\hline DT & Decision tree \\
\hline D2D & Device-to-device \\
\hline eMBB & Enhanced mobile broadband \\
\hline FOG & Freezing of gait \\
\hline FR1 & Frequency range one \\
\hline FR2 & Frequency range two \\
\hline GNSS & Global navigation satellite system \\
\hline IoT & Internet of Things \\
\hline KNN & K-nearest neighbor \\
\hline LOS & Line-of-sight \\
\hline LSTM & Long-short term memory \\
\hline MIMO & Multiple-input-multiple-output \\
\hline mMTC & Massive machine type communications \\
\hline mmWave & millimeter-wave \\
\hline NLOS & Non-line-of-sight \\
\hline NB & Naive Bayes \\
\hline OFDM & Orthogonal-frequency-division-multiplexing \\
\hline RRSS & received raw signal sample \\
\hline RSS & Received signal strength \\
\hline SNR & Signal-to-noise ratio \\
\hline SVM & Support vector machine \\
\hline ToA & Time-of-arrival \\
\hline ToF & Time-of-flight \\
\hline UE & User equipment \\
\hline URLLC & Ultra-reliable and low latency communications \\
\hline USRP & Universal software radio peripheral \\
\hline
\end{tabular}

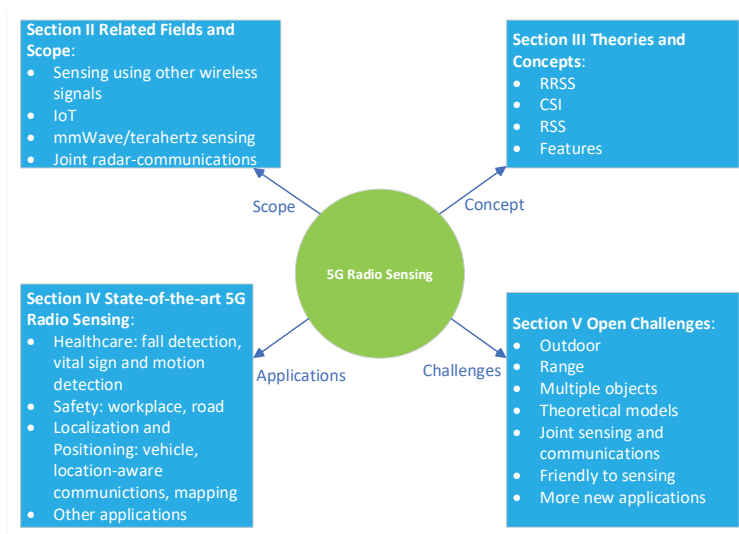

Fig. 2. Structure of the survey.

higher frequency and larger bandwidth, as discussed in Section I and Table I. In this sense, 5G sensing is distinct from radio sensing using WiFi or other wireless signals. Hence, this paper will only discuss $5 \mathrm{G}$ sensing.

The second field is IoT supported by 5G. A typical IoT framework is made of three main steps: data collection, data transmission and data processing. Among them, data transmission refers to the process of transmitting the field measurements from the remote nodes to the access point, and it can be implemented by using any wireless systems, including 5G. Indeed, one of the most important applications of $5 \mathrm{G}$ is 
mMTC that supports low-power wide-area networking for IoT. In this case, $5 \mathrm{G}$ provides the network for data transmission but is not involved in data collection. However, in $5 \mathrm{G}$ radio sensing, the $5 \mathrm{G}$ signal is used as the sensing signal to collect data. Hence, the two fields are different and this paper will not discuss any works on IoT supported by $5 \mathrm{G}$ network.

The third field is mmWave sensing. The similarity of the two fields comes from the fact that the 5G FR2 uses frequency bands in the mmWave spectrum. However, mmWave sensing has been adopted long before 5G systems were deployed, due to its high resolution [19]. For example, mmWave radar was used to acquire real-time visualization of 3D images [20] or see through walls [21]. This field is different from $5 \mathrm{G}$ radio sensing discussed here. First, mmWave sensing uses a mmWave waveform dedicated for sensing, while $5 \mathrm{G}$ radio sensing uses $5 \mathrm{G}$ waveforms designed for communications. For example, 5G signals use orthogonal-frequency-divisionmultiplexing (OFDM). Second, mmWave sensing in the literature uses specialist equipment, while $5 \mathrm{G}$ radio sensing uses existing $5 \mathrm{G}$ communications signals or devices for passive and opportunistic sensing. Hence, this paper will not discuss any works on mmWave sensing. Another related field is terahertz sensing. Similar to mmWave sensing, it uses active terahertz signals dedicated to imaging or sensing [22]. Recently, the interest in terahertz sensing grows due to the development of terahertz communications [23]. The terahertz band is expected to be an important enabler for the sixth generation [24]. Hence terhertz signals can be used for both sensing and communications [25]. However, 5G signals have not reached the terehertz band yet so this will not be discussed in the paper.

The last field is joint radar-communications for $5 \mathrm{G}$. Joint radar-communications has become important in recent years for two reasons: the lack of spectrum for communications and the demand for both radar sensing and data communications in emerging applications [26] - [28]. Radar and communications could co-exist, co-design, or integrate [26]. If they co-exist or co-design with each other, the radar sensing part of the joint system still uses radar waveforms for sensing and mutual interference between radar and communications exists. This can be mitigated via beamforming [31] - [33] or null space projection [34]. If they integrate with each other, dual-functional [35] or duplexed waveforms [29], [30] are often used, which require changes to the $5 \mathrm{G}$ systems. For example, the same carrier could be modulated by both radar and communications signals, or waveform diversity could be used where the main lobe is used for radar and side-lobes are used for communications, or certain antennas in MIMO systems or certain subcarriers in OFDM systems are used for radar while others are used for communications. The $5 \mathrm{G}$ radio sensing focuses on sensing only instead of joint designs. Hence, this paper will not discuss any works on joint radar-communications for $5 \mathrm{G}$.

In summary, this paper does not discuss any works on radio sensing using other wireless signals, IoT utilizing 5G network, sensing using dedicated mmWave signals, or $5 \mathrm{G}$ joint radar-communications. These fields are related but including them in this survey will make it excessively long. Moreover, there have already been survey papers on these relevant fields, such as [26] on joint radar-communications, and [18] on WiFi
TABLE III

SUMMARY OF DIFFERENCES OF RELEVANT FIELDS

\begin{tabular}{|c|l|}
\hline Relevant Field & \multicolumn{1}{|c|}{ Difference } \\
\hline Other wireless signals & $\begin{array}{l}\text { The unique characteristics of 5G systems, } \\
\text { such as high frequency, large bandwidth } \\
\text { and massive array, make 5G sensing a step } \\
\text { change compared with radio sensing using } \\
\text { WiFi and other cellular signals. }\end{array}$ \\
\hline IoT & $\begin{array}{l}\text { It uses the 5G network to transmit measure- } \\
\text { ments from remote sensors to the access } \\
\text { point but the 5G signal itself is not used } \\
\text { in data collection. }\end{array}$ \\
\hline mmWave/terahertz sensing & $\begin{array}{l}\text { It uses unmodulated waveforms and requires } \\
\text { specialist equipment for active sensing. }\end{array}$ \\
\hline Joint radar-communications & $\begin{array}{l}\text { It focuses on the interplay between radar } \\
\text { and communications and requires changes } \\
\text { to 5G systems. }\end{array}$ \\
\hline
\end{tabular}

sensing. To the best of the authors' knowledge, there have been no surveys on $5 \mathrm{G}$ radio sensing. For this reason and to fill the gap in the literature, this survey will only focus on $5 \mathrm{G}$ radio sensing. Next, we will introduce some basic theories and concepts used for signal processing in $5 \mathrm{G}$ radio sensing. Table III summarizes the difference of these fields from $5 \mathrm{G}$ radio sensing.

\section{THEORIES AND CONCEPTS}

Signals transmitted by one radio device and received by another radio device contain variations caused by objects in the ambient environment between the transmitter and the receiver. Hence, measurements of these signals can be used to sense the environment. There are several widely used types of measurements: received raw signal sample (RRSS), radio channel state information (CSI), received signal strength (RSS), or cellular signal quality (CSQ) indicator. RRSS is the raw sample of the received signal that directly reflects the signal variations. Hence, it contains all propagation characteristics. RRSS often uses signals received from references or beacons. CSI is a physical layer type of measurement to track the variations at the symbol level. It is able to discriminate multi-path components for high sensing accuracy. RSS is a link layer type to track the variations at the frame level. It is commonly used for link adaptation and transmission scheduling in wireless systems. CSQ is also a link layer type that is generated by mobile devices during idle mode to monitor radio link propagation from different base stations for possible cell reselection [8]. Other measurements include packet error rates, time-delay [36], Doppler [37] and link quality information [38].

\section{A. RRSS}

We discuss RRSS first. For $5 \mathrm{G}$ signals, assume that the OFDM waveform is used with a number of $N$ sub-carriers and a fixed frequency spacing of $\Delta f$. The transmitted signal after the inverse fast Fourier transform (IFFT) is

$$
S(t)=\sum_{n=1}^{N} s_{n}(t)=\sum_{n=1}^{N} A_{n} e^{j 2 \pi n \Delta f t},
$$


where $s_{n}(t)$ is the signal on the $n$-th sub-carrier and $A_{n}=$ $a_{n} e^{j \theta_{n}}$ is the transmitted data symbol on the $n$-th sub-carrier with amplitude $a_{n}$ and phase $\theta_{n}$.

This signal is transmitted in an environment with $K$ objects. The RRSS on the $n$-th sub-carrier after going through the environment is given by

$$
\begin{aligned}
Y_{n}(t) & =\sum_{k=1}^{K} \alpha_{k} A_{n} e^{j 2 \pi n \Delta f\left(t-\tau_{k}\right)} e^{j 2 \pi f_{k} t}+W_{n}(t) \\
& =\sum_{k=1}^{K} \alpha_{k} e^{j 2 \pi f_{k} t-j 2 \pi n \Delta f \tau_{k}} s_{n}(t)+W_{n}(t),
\end{aligned}
$$

where $\alpha_{k}$ is the effective attenuation from the $k$-th object, $\tau_{k}$ is the time delay of the $k$-th object, $f_{k}$ is the Doppler of the $k$-th object if it is moving, and $W_{n}$ is the white noise.

In practice, more than one OFDM symbols will be used. Denote $T_{s}=\frac{1}{\Delta f}+T_{c p}$ as the interval of the OFDM symbol, where $T_{c p}$ is the length of the cyclic prefix. Thus, by substituting $t=m T_{s}$ in (2), one has the received $m$-th OFDM symbol on the $n$-th sub-carrier as

$$
Y_{m, n}=\sum_{k=1}^{K} \alpha_{k} e^{j 2 \pi f_{k} m T_{s}-j 2 \pi n \Delta f \tau_{k}} s_{m, n}+W_{m, n},
$$

where $m=1,2, \cdots, M$ index the OFDM symbols, $W_{m, n}=$ $W_{n}\left(m T_{s}\right)$ and $s_{m, n}=s_{n}\left(m T_{s}\right)$. According to the radar range equation, one has $\left|\alpha_{k}\right|^{2}=\frac{G_{t} G_{r} \lambda^{2} \sigma_{k}}{(4 \pi)^{3} d_{k}^{4}}$, where $G_{t}$ and $G_{r}$ are the transmitting and receiving antenna gains, respectively, $\lambda$ is the wavelength of the signal, $\sigma_{k}$ is the mean radar cross section related to the reflection area of the object, and $d_{k}$ is the distance between the transmitter and the $k$-th object in the case when the transmitter and receiver are collocated. Thus, the attenuation is determined by the range or the distance. One sees from (3) that the time delay $\tau_{k}$ causes different phase shifts for different sub-carriers, and the Doppler causes different phase shifts for different OFDM symbols.

If the transmitted symbol is known, such as references or beacons, one has

$$
Q_{m, n}=\frac{Y_{m, n}}{s_{m, n}}=\sum_{k=1}^{K} \alpha_{k} e^{j 2 \pi f_{k} m T_{s}-j 2 \pi n \Delta f \tau_{k}}+W_{m, n}^{\prime}
$$

where $W_{m, n}^{\prime}=\frac{W_{m, n}}{s_{m} n}$ and $Q_{m, n}$ contains the information on the objects. This RRSS can be used for radio sensing.

For example, if ranging and localization are performed, one can calculate the periodogram as the range-Doppler profile to give [39] $P(i, k)=$ $\left|\sum_{m=0}^{M^{\prime}-1} e^{-j 2 \pi \frac{i m}{M^{\prime}}}\left(\sum_{n=0}^{N^{\prime}-1} Q_{m, n} e^{j 2 \pi \frac{n k}{N^{\prime}}}\right)\right|^{2}$, where $M^{\prime} \geq M$ and $N^{\prime} \geq N$. Using the range-Doppler profile, the presence of the object is detected by comparing $P(i, k)$ with a predetermined threshold. Ranging and localization are important applications of $5 \mathrm{G}$ radio sensing and they often use RRSS measurements. The ranging resolution is given by $\Delta d=\frac{c}{2 B}$ and the velocity resolution is given by $\Delta v=\frac{c}{2 T_{f} f_{c}}$, where $c$ is the speed of light, $B$ is the channel bandwidth, $T_{f}$ is the time duration of one frame and $f_{c}$ is the carrier frequency [39]. Fig. 3 shows how they change with bandwidth and carrier frequency, respectively, when $T_{f}=5 \mathrm{~ms}$. Since
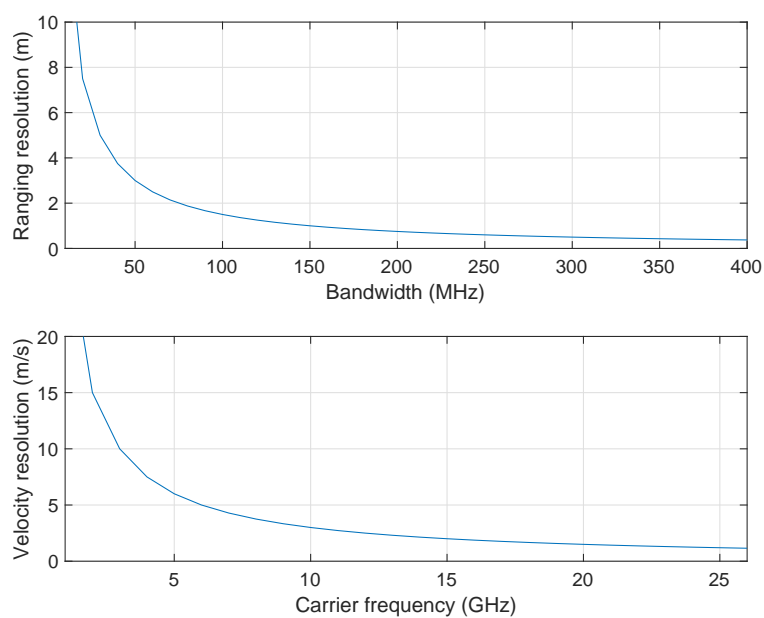

Fig. 3. Ranging resolution versus bandwidth and velocity resolution versus carrier frequency.

$5 \mathrm{G}$ signals have a bandwidth of up to $400 \mathrm{MHz}$ and carrier frequency of $26 \mathrm{GHz}$, it has great advantages in ranging and localization over WiFi signals.

\section{B. CSI}

CSI is another widely used type of measurement for $5 \mathrm{G}$ radio sensing. Assume that the CSI representing the static ambient environment is given by

$$
h(\beta, \phi \mid \emptyset)=\sum_{p=1}^{P} \beta_{p \mid \emptyset} e^{-j \phi_{p \mid \emptyset}}
$$

where $P$ is the total number of multi-path components, $\beta_{p \mid \emptyset}$ is the gain of the $p$-th component, $\phi_{p \mid \emptyset}$ is the phase of the $p$-th component and $\emptyset$ represent the channel state in the static case with no changes or events happening. Note that the model in (5) describes the multi-path components in the channel, while the model in (2) describes the reflections caused by multiple objects in the ambient environment. They are related to each other but not necessarily the same.

When one or more objects enter the environment or perform activities, such as a car driving into a street or a human in a room raising the arm, the state of the channel will change due to these changes or events. In this case, the CSI becomes

$$
h_{n}(\beta, \phi \mid \amalg)=\sum_{p=1}^{P} \beta_{p \mid \amalg}^{n} e^{-j \phi_{p \mid \amalg}^{n}},
$$

where $n=1,2, \cdots, N$ denote different time instants, $\beta_{p \mid \amalg}^{n}=$ $\beta_{p \mid \emptyset}+\Delta \beta_{p \mid \amalg}^{n}, \phi_{p \mid \amalg}^{n}=\phi_{p \mid \emptyset}+\Delta \phi_{p \mid \amalg}^{n}, \Delta \beta_{p \mid \amalg}^{n}$ and $\Delta \phi_{p \mid \amalg}^{n}$ are the amplitude and phase changes induced by event $\amalg$ at time $n$ for the $p$-th multi-path component, and $\amalg$ represents the channel state with event $\amalg$. The amplitude change and the phase change contain information on the event. In practice, the amplitude information is often used for sensing. However, the amplitude information is suitable for detection but not for discrimination of different activities. Thus, other applications use phase or both amplitude and phase for sensing. 
When multiple carriers or multiple antennas are used, the CSI is similar, except that frequency diversity gain or spatial diversity gain will be achieved by having independent measurements on different frequencies or antennas. In this case, (5) and (6) become vectors. They are not repeated here for compactness.

\section{RSS}

The RSS can be expressed as

$$
S_{n}(\amalg)=S(\emptyset)+\Delta S_{n}(\amalg),
$$

where $S(\emptyset)=E\left\{S_{T}(\emptyset)\right\}$ is the average RSS observed over a certain period of time $T$ when the channel is in static state with no events and $\Delta S_{n}(\amalg)$ is the additional RSS induced by event $\amalg$ at time instant $n$. For IEEE 802.15.4, the RSS indicator (RSSI) can be used with an 8-bit resolution. For WiFi, RSS can be obtained using the received channel power indicator (RCPI). For 4G systems, RSS can be obtained from the reference signal received power (RSRP). For $5 \mathrm{G}$, there are several RSS that can be used: synchronization signal reference signal received power (SS-RSRP), new radio received signal strength indicator (NR-RSSI) etc. Activity and behavior recognition is an important part of healthcare monitoring applications using $5 \mathrm{G}$ radio sensing, and they often use CSI and RSS. In this case, machine learning is often applied.

\section{Features of Measurements for Machine Learning}

In machine learning, features are often calculated and selected from the raw measurements before learning. This subsection introduces commonly used features in $5 \mathrm{G}$ sensing. Assume a window size of $W$. Commonly used features include the following. The mean or average of the measurements is $\mu=\frac{1}{W} \sum_{w=1}^{W} d_{w}$, where $d_{w}$ represents either RSS, CSI or RRSS discussed in the previous subsections. The variance of the measurements is $\sigma^{2}=\frac{1}{W} \sum_{w=1}^{W}\left(d_{w}-\mu\right)^{2}$. The $n$ order central moment is $\gamma=\frac{1}{W} \sum_{w=1}^{W}\left(d_{w}-\mu\right)^{n}$. These are time-domain features of the received radio signal. Assume $D_{q}=\sum_{w=1}^{W} d_{w} e^{-j \frac{2 \pi}{Q} q w}$ as the FFT of $d_{w}$, where $q=$ $1,2, \cdots, Q$. The spectral energy is [40] $E=\sum_{q=1}^{Q}\left|D_{q}\right|^{2}$. Let $P_{q}=\frac{\left|D_{q}\right|^{2}}{\sum_{q=1}^{Q}\left|D_{q}\right|^{2}}$. The entropy is $H=\sum_{q=1}^{Q} P_{q} \ln P_{q}$. These are the frequency domain features.

Other features include root mean square, median, minimum, maximum, standard deviation, auto-correlation, zero crossing rate, Kurtosis, and skewness.

\section{E. Downlink- and Uplink-based Sensing}

Downlink-based sensing uses the $5 \mathrm{G}$ signals transmitted by the base station, while uplink-based sensing uses the $5 \mathrm{G}$ signals transmitted by the user equipment (UE). Downlinkbased sensing has long range and large coverage but the propagation environment is more complicated due to more interference and objects. Uplink-based sensing has short range and small coverage but the propagation environment is less complicated due to the short range. Most works using RRSS and CSI are downlink-based, while those using CSQ are uplink-based.

\section{State-OF-ThE-ART 5G RAdio SENSING}

$5 \mathrm{G}$ radio sensing extracts important information about the ambient environment from the received $5 \mathrm{G}$ signals. These information can be human-centric. For example, 5G radio sensing recognizes the presence and motion of humans [41], [42], [43], the fall [44], [45], gait [46], walking speed [47], [48], gesture, vital signs [49], [50]. These information can be object-centric. For example, $5 \mathrm{G}$ radio sensing can detect the presence and motion of objects to localize and track objects [52] - [57], and image the objects [58]. These information can be scene-centric. For example, $5 \mathrm{G}$ radio sensing can count the crowd size or perform mapping and positioning [59] - [61].

The extracted information has enabled a wide variety of applications using $5 \mathrm{G}$ radio sensing. For example, the presence and motion detection of humans can be used for human safety in industrial workspace or on the roads for safety and surveillance [62] - [71]. The human fall and emotion detection can be used for ambient assisted living, the human gait, sleep and vital sign monitoring for patient recovery or treatment, the gesture recognition for home automation to provide context-aware services, while the human activity and behavior recognition for intrusion detection. The detection of objects can be used for material identification [73], [74]. The localization and tracking of objects can be used in warehouses and see-through-walls imaging. The crowd counting can be used in social mass gathering for security and surveillance or for heating, ventilation and air-conditioning controls. The indoor positioning and mapping can be used to map the layout of a room [75] - [79].

Next, we will discuss the state-of-the-art works on $5 \mathrm{G}$ radio sensing based on their applications, as most of these works are application-oriented. Nevertheless, this categorization may lead to cross-overs between applications and we will minimize the cross-overs in the discussion.

\section{A. Healthcare}

$5 \mathrm{G}$ radio sensing used in healthcare helps to analyze and understand human behavior or patient's daily activities and syndromes to improve reliability of diagnosis and decrease working load for healthcare workers.

1) Fall Detection: Fall detection is one of the most important applications of $5 \mathrm{G}$ radio sensing in healthcare. It is critical for life-or-death and cost reasons, especially for elderly people who live alone or for vulnerable people who suffer from certain diseases. Without prompt response and emergency care, the fall could develop into life-threatening conditions. Timely detection of a fall is thus of great importance. There has been a large amount of literature on fall detection (see [44] and references therein but they mainly use WiFi sensing), and $5 \mathrm{G}$ radio sensing can play an important role with its merits.

In [45], the authors studied fall detection using the $5 \mathrm{G}$ C-band (3.3 - 4.2 GHz, 4.4 - $5.5 \mathrm{GHz}$ ) for patients after surgeries. The patients after surgeries are often vulnerable. A fall could lead to many injuries or even to death, such as bruises, bone fractures, neurological disorders or reopening of wounds. Reference [45] provided a low-cost solution. They used a Rhode \& Schwartz SMBV 100B signal generator 
to generate $5 \mathrm{G}$ signals at a frequency of $4.8 \mathrm{GHz}$ and a transmission power of $20 \mathrm{dBm}$, which was radiated using an omnidirectional antenna. The CSI was received by an ASUS PCE-N15 NIC connected to a desktop PC. The fall, slow walking, fast walking, lying, squatting and sitting were monitored from 8 subjects of different ages located 4 meters away from both the transmitter and the receiver. The CSI measurements were organized in packets from 30 sub-carriers and eventually the 15-th sub-carrier was chosen due to its good accuracy. Three machine learning algorithms were used to process data: support vector machine (SVM), Naive Bayes (NB) and decision tree (DT). The experiments showed that SVM has the best performance. Tests using three kernels of polynomial, linear and radial basis function (RBF) showed that RBF has the best performance for SVM. The highest accuracy reaches more than 95\%. The Kappa value or Cohen's Kappa score compares the expected accuracy with the observed accuracy for a classifier [80]. The closer it is to 1, the better the classifier will be. In this case, the Kappa value for SVM is 0.98 , very close to the upper limit of 1 .

2) Vital Sign and Motion Detection: Vital signs, such as respiration rate, heart rate or pulse rate, are direct indicators of health. Gait and other motions have also been shown as an important indicator and predictor of the health status, especially in the elderly.

In [49], the authors studied the use of 5G C-band signals operating at $4.8 \mathrm{GHz}$ to detect the tremors and breathing activities for multiple sclerosis (MS) patients. MS is a motion-related neuro-degenerative disease. It causes mobility impairment and many functional disabilities. One syndrome of this disease is tremor due to muscle weakness, spasm and ataxia, which could lead to paralysis. Another syndrome related to this is the lost control of breathing, due to weak respiratory muscles. The authors used the same equipment as [45] to capture CSI at 4.8 $\mathrm{GHz}$ from 30 sub-carriers. Three machine learning algorithms were used: SVM, K-nearest neighbor (KNN) and random forest $(\mathrm{RF})$. Their performances were examined in terms of accuracy, precision, recall, specific, Kappa and F-measure. Four motions: sitting, lying, falling and tremors, were detected, where the transmitter and receiver were 4 to 5 meters away. Breathing detection was performed when they were 1 to 1.5 meters away, and compared with a respiratory sensor. Features used include root mean square, square root of amplitude, mean, Kurtosis, crest factor, skewness, impact factor, peak-topeak, standard deviation, marginal factor. Overall, SVM has the best performance with an accuracy of $95 \%$ for sitting, $98 \%$ for lying, $100 \%$ for falling and $96 \%$ for tremor, and its Kappa value is 0.96 . Its recall, precision, specificity and F-measure values are also higher. For breathing detection, the correlation between $5 \mathrm{G}$ sensor and a wearable respiratory sensor is 0.88 for normal breathing and 0.87 for abnormal breathing, showing the effectiveness of $5 \mathrm{G}$ sensing. The same authors also worked on body motion detection for Huntington disease (HD) [42]. HD commonly affects people in their thirties and forties. The most common symptom is chorea, where patient feels uncontrollable jerky moving or body twitching. Monitoring body motion is therefore essential to discover and treat HD. They focused on SVM to give an accuracy of $96 \%$ to $99 \%$.
In [50], the authors proposed a breathing monitoring system using $5 \mathrm{G} \mathrm{C}$-band at $4.8 \mathrm{GHz}$ to capture breathing-induced chest movements in diabetic ketoacidosis (DKA) patients. Typical signs of DKA include Kussmaul breathing, a deep and labored breathing pattern, and a fruit-scented breath. Their experiments showed that, in normal breathing, the respiratory rate is 17 breaths/minute and extent is between -1 and 1 . In Kussmaul breathing, the rate is 32 breaths/minute and extent is larger than 1 . Thus, the respiratory rate and extent were used to diagnose and monitor DKA. The amplitude of the CSI was used and the data was first pre-processed using wavelet transform to remove outliers and then peak detection was used to calculate respiratory rate and extent. The distance between transmitter and receiver was 2 to 3 meters. Ground truth was obtained using HKH-11C digital respiratory sensor. Out of 30 sub-carriers, the 13-th was chosen as the best. SVM was used to classify normal breathing or Kussmaul breathing. The accuracy is $98.75 \%$.

In [46], the authors used 5G C-band signals to detect the freezing of gait (FOG) episodes experienced by Parkinson's disease (PD). They used a signal generator DSC3000 series, two dipole antennas, TP-link (PCE-AC68) NIC, and a desktop to capture the amplitude of CSI, which was then converted into time domain features of mean, standard deviation, skewness, Kurtosis, mean absolute deviation, interquartile range and peaks. Then, these features were used in a multi-layer perception neural network with a single input layer, single hidden and output layer using Levenberg-Marquardt training to classify between sitting, slow walk, fast walk, voluntary stop and FOG episodes. Details of these neural network methods can be found in [81]. The classification for FOG is $99.3 \%$, better than any existing methods.

In summary, 5G radio sensing has great potential in healthcare applications to save and protect lives. It allows the identification of key symptoms of various diseases that are related to human body movements. The identification often uses the CSI or the amplitude of CSI. It requires comparison between normal people and patients for training. The classification is performed by using machine learning. SVM has the best overall performance in many cases so far. Neural networks offer an alternative. Table IV summarizes the application of 5G radio sensing in healthcare. Several lessons are learned. Firstly, it is observed from [46] that the neural networks methods can greatly improve the accuracy of $5 \mathrm{G}$ radio sensing. However, very few works used these methods. Thus, the detection in [45], [49], [42], [50] could be further improved by using neural networks, at the cost of much longer data records for training. Secondly, these works overwhelmingly used CSI. However, as discussed in Section III, RRSS contains more information about the objects and could be used to improve accuracy. Finally, most of these works used the $4.8 \mathrm{GHz}$ band. Higher $5 \mathrm{G}$ frequencies could be used to improve performance. For healthcare applications, detection accuracy is critical.

\section{B. Safety}

The ultimate goal of safety is to protect lives from danger. In this case, $5 \mathrm{G}$ radio sensing can acquire an environmental 
TABLE IV

HEALTHCARE APPLICATIONS OF 5G RADIO SENSING

\begin{tabular}{|c|c|c|c|c|c|c|c|c|}
\hline Ref. & Diseases & Detectors & Frequency & Bandwidth & Antenna & Metrics & Methods & Accuracies \\
\hline$[45]$ & post-surgical patient & fall & $4.8 \mathrm{GHz}$ & 30 sub-carriers & single omni & CSI & SVM, NB, DT & $>95 \%$ \\
\hline [49] & multiple sclerosis & tremor, breath, & $4.8 \mathrm{GHz}$ & 30 sub-carriers & single omni & CSI & SVM, KNN, RF & $\begin{array}{l}>95 \% \\
\text { for } \\
\text { tremor, } \\
0.88 \\
\text { correla- } \\
\text { tion for } \\
\text { breath- } \\
\text { ing }\end{array}$ \\
\hline$[42]$ & Huntington disease & body motion & $4.8 \mathrm{GHz}$ & 30 sub-carriers & single omni & CSI & SVM & $96 \%$ to $99 \%$ \\
\hline$[50]$ & Diabetic ketoacidosis & breathing & $4.8 \mathrm{GHz}$ & 30 sub-carriers & single omni & amplitude of CSI & SVM & $98.75 \%$ \\
\hline [46] & Parkinson's disease & freezing of gait & $4.8 \mathrm{GHz}$ & 30 sub-carriers & single omni & amplitude of CSI & neural networks & $99.3 \%$ \\
\hline
\end{tabular}

perception and use this perception to make critical decisions on how humans should act and behave within safe limits.

1) Workplace Safety: In a smart factory, humans often work together with robotics, such as on a car assembly line. In this case, $5 \mathrm{G}$ radio sensing can perform human activity recognition to enhance the transparency of human-machine interaction. This allows a robot to follow human movements without obstructing human operations. For example, near hazardous machinery, a virtual fence could be built by using $5 \mathrm{G}$ radio sensing so that an alert will be made if humans get too close.

To realize this, the non-cooperative detection of human presence is important so that human operations will not be disrupted. Human activity recognition is also important. One area related to human activity detection is human gesture and pose detection. Also, human activity recognition in surveillance systems tracks individuals and crowds to support the security guard to monitor suspicious activities and detect possible hazards or anomalies.

In [8] and [43], the authors used CSQ measurements from mobile devices, including $5 \mathrm{G}$ networks, to detect body movements near smartphone. Even in idle mode, smartphone carries out continuous and autonomous measurements of the downlink channels from different base stations for cell reselection. Any fluctuation in these measurements reflects body movement or scene change in the surrounding. In these works, when the body is less than 0.5 meter away from the device, CSQ measurements were taken for one month from 2 to 4 smartphones, and then processed using a sequential change detection algorithm based on cumulative sum to isolate changes due to body motion from changes due to network. Then, machine learning was applied to these detected changes using Bayesian classification based on Dirichlet-Compound Multinomial model, SVM, and long-short term memory (LSTM), to identify the body motion. Off-the-shelf smartphones were used. SVM has an accuracy of $80 \%$, Bayesian has an accuracy of $90 \%$, while LSTM has comparable performance with Bayesian.

In [5], 5G sensing was used for workplace safety. For a typical human-robot collaborative scenario with one robot handling tasks and located inside an open workspace that is shared with one human worker. Both were moving so that the position of human must be tracked continuously. The authors studied three methods: WiFi passive sensing with 16 industrial standard wireless nodes, fast beam-steering and passive sensing using $100 \mathrm{GHz}$. In the last case, a virtual safety fence was implemented to discriminate safe and unsafe activities within 0.5 to $2 \mathrm{~m}$ of the robots. CSI measurements were used in maximum likelihood estimation, LSTM, and a deep neural network. The deep neural network achieves an accuracy of $99 \%$ for recognizing standing, moving arms and crossing fence. A similar study was done in [64]. Considering a human-robot collaborative workspace, a mixture of 6 frequency modulated continuous wave radars in sub-THz band, 3 infrared sensors, one radio transmitter for opportunistic sensing at $100 \mathrm{GHz}$, and a sub-THz camera, with 1024 detectors, were deployed to detect and track human operators in a virtual fence system. CSI was used. The classification accuracy is larger than $96 \%$ with a latency less than $150 \mathrm{~ms}$.

In [41], the authors used a prototype $5 \mathrm{G}$ system with 52 OFDM sub-carriers and a large sub-carrier spacing of 240 $\mathrm{kHz}$ at a center frequency of $3.45 \mathrm{GHz}$ to detect presence and walking speed. In presence detection, two universal software radio peripheral (USRP) devices (USRP X310) were used as transmitter and receiver in two rooms, a TP-LINK omnidirectional antenna with a gain of $18 \mathrm{dBi}$ for both transmitter and receiver and a window of $60 \mathrm{~ms}$ equivalent to $300 \mathrm{OFDM}$ symbols. In walking speed detection, two USRP NI 2932 devices were used as transmitter and receiver. The transmitter used TP-LINK omni-directional antenna with $18 \mathrm{dBi}$, while the receiver used a planar antenna with $5 \mathrm{dBi}$. They were 20 meters away from each other. A window of $20 \mathrm{~ms}$ was used. They used both time and frequency domain features, including mean, standard deviation, variance, root mean square, frequency spectral entropy, Kurtosis, skewness from the RSS measurement applied to KNN. The best accuracies were $92.8 \%$ and $95 \%$ for presence and walking speed, respectively, in comparison with $70 \%$ by using a sensor. References [47] and [48] give more details on walking speed recognition performed in [41]. A similar study was done in [62].

Reference [65] studied the use of beam-steering to recognize distinct activities from multiple humans. This task is much more challenging than recognition of a single human but is necessary in workplaces where multiple human operators work with robots. They used receiver-side beamforming and beam-sweeping over different azimuth angles to detect human presence. Beamforming is very energy-consuming and hence, will not be constantly available in the environment, unless the receiver is customized for sensing instead of using existing $5 \mathrm{G}$ terminals. Thus, this method has limited use. The experiment 
used the sub- $6 \mathrm{GHz}$ band and a USRP X300 with one antenna as transmitter and three USRPs as receivers each with phase synchronized 4 antennas and having 52 OFDM sub-carriers in a $22.4 \mathrm{~m}^{2}$ semi-anechoic chamber. The method can detect 0 to 4 persons within one person error in 100 experiments, 5 persons in 43 out of 100 experiments, 6 persons in 50 out of 100 experiments, without any prior training. It can also extract 20 gestures out of 21 from one person when up to three were simultaneously there. CSI was used. A similar study was performed in [63] and [66], where receiver-side beamforming was able to detect the direction-of-arrivals for up to four stationary humans and track the direction-of-arrival of up to two walking humans at the same time.

2) Road Safety: Road safety is another important application of $5 \mathrm{G}$ radio sensing. This is particularly useful because $5 \mathrm{G}$ is chosen as the backbone to support vehicle-to-everything communications and hence, future vehicular networks are likely to be built within $5 \mathrm{G}$ networks to use ambient $5 \mathrm{G}$ signals for road sensing [67]. With the advent of autonomous driving, this application becomes critical, as driver-less cars need to sense the driving environment not only for efficiency, such as route planning and energy saving, but also for safety to protect other road users as well as avoid any accidents. Traditional methods often adopt radars, lidars, cameras or sonars. However, most of these methods require LOS, which is not possible in some cases. $5 \mathrm{G}$ radio sensing could be a useful complement to active radars.

In [71], a detector for crossing a curb was proposed to protect pedestrians, as they may be crossing the road between two parked cars without being noticed by drivers on the road. First, a context filter based on human activity recognition was designed to detect the pedestrian's position, movement direction and acceleration to determine if the pedestrian is stepping on the road. Then the result will be communicated to the nearby cars via $5 \mathrm{G}$ internet and D2D communications. The study used sensor data from smartphones carried by pedestrians but it could also use $5 \mathrm{G}$ signals from road side units. In the detection, the curb crossing is made of three steps: standing at or walking to the curb, crossing the curb and standing or walking towards the road. Three curb heights were tested based on German standards. Five activities were considered from standing, slow walking, fast walking, running to crossing. The accelerometer and gyroscope were used to acquire data. Machine learning algorithms including KNN, C4.5, RIPPER2, NB, sequential minimal optimization (SMO) were applied to features of mean, variance, standard deviation, energy, entropy and integral. KNN has the best overall performance of a missed alarm rate between $19 \%$ and $33 \%$. In [72], the authors focused on the recognition of crossing curb or stepping down alone. They proposed a frequency domain method to analyze the frequency components of the data followed by low-pass and high-pass filters, and a grouping method to improve recognition by grouping similar activities in the data. The new method improved the F-measure by $20 \%$, precision by $18.7 \%$ and recall by $20.9 \%$, compared with state of the art method, for crossing curb recognition. A detailed discussion of pedestrian safety was given in [70].

In [61], the authors proposed a new concept of cooper- ative passive coherent location (CPCL) scheme to support road safety using $5 \mathrm{G}$ radio sensing. Its novelty is to extend traditional passive radar using one node to cooperative passive radar involving several nodes. In CPCL, any radio acts as an illuminator or observer. Locally estimated object parameters were exchanged and fused, creating a powerful radar network. The authors discussed possible issues in radar signal processing, networking, synchronization, and data fusion. Finally they showed an initial example using one transmitter and two receivers to prove that cooperation is beneficial.

In [68], the authors proposed a new road surveillance system using 5G OFDM signals. The surveillance was enabled by using the combination of delay and Doppler estimates from multiple bistatic passive radar links. The Cramer-Rao lower bounds for joint estimation of delay and Doppler were derived. A parameter set for low signal-to-noise ratio (SNR) applications was obtained to satisfy the accuracy requirement while reducing the bandwidth requirement on delay estimation. The minimum required observation time was also determined for Doppler estimation.

In summary, 5G radio sensing has an important use in road safety. This is driven by the adoption of $5 \mathrm{G}$ networks in future vehicular networks for smart traffic as well as the pervasiveness of smart phones. The targets of sensing in this use are pedestrians to protect vulnerable road users and vehicles for road surveillance. However, due to the complexity on the road, this sensing is challenging and therefore, requires cooperation sometimes to improve accuracy and reliability. Table $\mathrm{V}$ summarizes the applications of $5 \mathrm{G}$ radio sensing to enhance safety. The lessons learned can be summarized as follows. Comparing these works, it is concluded that high frequency leads to high accuracy, as in [5] and [64]. Thus, other works could also explore the high frequency to achieve better accuracy. Also, the foundation of these applications is motion detection. Motion detection requires the decomposition of the whole process into several typical stances. This decomposition is the key and could seriously affect the sensing accuracy.

\section{Localization and Positioning}

Localization and positioning are important applications of radio sensing. There have been a lot of works on devicefree localization even before $5 \mathrm{G}$ was deployed [69]. The deployment of $5 \mathrm{G}$ systems makes these applications extremely attractive, as 5G radio sensing can achieve an accuracy of centimeters, a much better result than the commonly used GNSS-based method with an accuracy of several meters. In $5 \mathrm{G}$ radio sensing, the localization is device-free or sensor-less.

1) Vehicle Positioning: Similar to road safety, vehicle localization is an important application of 5G sensing, because the vehicular networks are inherently built within the $5 \mathrm{G}$ networks. For autonomous driving, finding where the vehicle is determines all the following actions, such as route planning and traffic monitoring. 5G signals have many unique characteristics that make them suitable for vehicle positioning.

In [15], a feasibility study was performed to use the 5G communications waveforms as radar signals. Different $5 \mathrm{G}$ frequencies and bandwidths were discussed. Three scenarios 
TABLE V

SAFETY APPLICATIONS OF 5G RADIO SENSING

\begin{tabular}{|c|c|c|c|c|c|c|c|}
\hline Ref. & Application & Detectors & Frequency & Bandwidth & Metrics & Methods & Accuracies \\
\hline [8], [43] & surrounding & body movement & unknown & unknown & CSQ & $\begin{array}{l}\text { SVM, } \\
\text { DCM, } \\
\text { LSTM }\end{array}$ & $90 \%$ \\
\hline$[5]$ & human-robot & standing, arm-moving, crossing & $100 \mathrm{GHz}$ & unknown & CSI & $\begin{array}{l}\text { MLE, } \\
\text { LSTM, } \\
\text { Deep } \\
\text { Neural }\end{array}$ & $99 \%$ \\
\hline [64] & virtual fence & body motion & $100 \mathrm{GHz}$ & unknown & CSI & $\begin{array}{l}\text { machine } \\
\text { learning }\end{array}$ & $96 \%$ \\
\hline$[41],[47],[48],[62]$ & workspace & presence, walking speed & $3.45 \mathrm{GHz}$ & $12.48 \mathrm{MHz}$ & RSS & $\mathrm{KNN}$ & $92.8 \%-95 \%$ \\
\hline$[65],[63],[66]$ & workspace & $\begin{array}{l}\text { presence and ac- } \\
\text { tivity from multi- } \\
\text { ple }\end{array}$ & $3.42 \mathrm{GHz}$ & $12.48 \mathrm{MHz}$ & CSI & $\begin{array}{l}\text { MMSE } \\
\text { beamform- } \\
\text { ing }\end{array}$ & high \\
\hline$[71],[72]$ & pedestrian & crossing curb & unknown & unknown & $\begin{array}{l}\text { accelero- } \\
\text { meter } \\
\text { and gy- } \\
\text { roscope }\end{array}$ & $\begin{array}{l}\text { KNN, } \\
\text { C4.5, } \\
\text { RIPPER2, } \\
\text { Bayes, } \\
\text { SMO }\end{array}$ & $80 \%$ \\
\hline$[61]$ & road safety & car locations & unknown & $80 \mathrm{MHz}$ & RRSS & $\begin{array}{l}\text { Bayesian } \\
\text { fusion and } \\
\text { MEC }\end{array}$ & unknown \\
\hline$[68]$ & road surveillance & range and velocity & $5.2 \mathrm{GHz}$ & $10 \mathrm{MHz}$ to $1 \mathrm{GHz}$ & RRSS & $\begin{array}{l}\text { Delay, } \\
\text { Doppler } \\
\text { estimation }\end{array}$ & unknown \\
\hline
\end{tabular}

of $5 \mathrm{G}$ radar in vehicle radar, marine radar and aviation radar were described. For adaptive cruise control, a range resolution of less than 0.5 meter is required and can be satisfied using a bandwidth of $400 \mathrm{MHz}$ as in $5 \mathrm{G}$ new radio. The related operation ranges for radar and communications are 90 meters and 150 meters, respectively, which could be further increased by increasing the transmission power within FCC limits. Also, a SNR higher than $15 \mathrm{~dB}$ is required to satisfy the velocity resolution requirement. A joint receiver for radar parameter estimation and timing synchronization was also proposed and the simulation of mean squared errors showed that coarse range and velocity estimates can be achieved.

In [51], 5G mmWave communications signals were proposed for vehicle positioning to replace GNSS-based positioning. GNSS is limited by blockage, and it treats multipath as interference, while $5 \mathrm{G}$ localization utilizes multi-path for simultaneous localization and mapping based on time of arrival measurements. Assuming both reflecting surfaces and scattering points, the work proposed a Bayesian 5G tracking filter followed by a brief propagation filter. The tracking filer performed data association to match paths to map entries. The propagation filter computed posterior distributions over vehicle state and map entries, and distinguished different sources without prior knowledge. This algorithms gave an average tracking error of 0.5 meters, 0.02 radians, and 0.3 meters for the vehicle location, heading and clock bias, respectively. Reference [59] gave an overview of vehicle positioning using $5 \mathrm{G}$ signals.

In [54], the authors studied the use of 5G signals transmitted by the base station as a monostatic radar for ranging in a time- division multiplexing systems. First, they analyzed the range and velocity estimation resolutions for different bandwidths and observation time. 5G FR1 and 5G FR2 can give a range resolution of around 1.5 meters and 0.4 meters, respectively. The best option is FR2 with bandwidth $400 \mathrm{MHz}$ and subcarrier spacing $120 \mathrm{kHz}$. For the velocity resolution, FR2 is an order of magnitude better than FR1. In their experiments, they used a NI PXIe-5840 vector signal transceiver as transmitter and receiver at $3.5 \mathrm{GHz}$ and then two Keysight N5183BMXG signal generators to up-convert and down-convert them to $28 \mathrm{GHz}$. The system verified the proposal for indoor and outdoor sensing. A similar study was also performed in [60] by the same authors with more emphasis on self-interference removal.

2) Location-aware Communications: The UE location is important in cellular communications for many location-based services, but it is even more important in 5G systems, as it can improve the radio access network, for example, by enabling location-based beamforming to reduce interference and strengthen signals or location-based mobility and radio resource management to improve spectral efficiency. If the UE is the vehicle, then the methods in the previous subsection may also be used for UE positioning and tracking.

In [53], the advantages of UE positioning in 5G were first reviewed. Then, using LOS, an extended Kalman filter was used to track its directional parameters for positioning performed at the base station. A ultra-dense network with 74 access points (AP) was used. The APs use circular arrays with 20 elements and transmission power of $23 \mathrm{dBm}$. The UE uses circular arrays with 4 elements and transmission power of 10 
$\mathrm{dBm}$. The UE travels between $30 \mathrm{~km} / \mathrm{h}$ to $50 \mathrm{~km} / \mathrm{h}$. The system operated at $3.5 \mathrm{GHz}$ with OFDM for both up and down links. Sub-carrier spacing is $240 \mathrm{kHz}$ and transmission interval is 200 micro-seconds using Zadoff-Chu sequence. Both centralized and distributed tracking were performed. The error in azimuth angle at AP is less than 0.1 degrees and in elevation angle at AP is less than 3 degrees, in all cases. Tracking at UE has larger errors. The authors also compared traditional CSI-based beamforming with location-based beamforming and showed that receiver side location-based beamforming has excellent throughput performances. This saves the overhead for channel estimation, which is a serious issue in massive MIMO due to pilot contamination.

In [55], the authors studied different approaches of the UE positioning for mmWave communications using LOS, NLOS, combined LOS and NLOS. Using a transmission power of 10 $\mathrm{mW}$ and a frequency of $60 \mathrm{GHz}$, trilateration methods using RSS, ToF and AoA were compared. It was shown that RSS is generally poor, while ToF and AoA have good performances with LOS, with possible more improvement by combining ToF and AoA. For small spaces, combining LOS with the 1st order reflection signals and using both ToF and AoA give decimeter level accuracy. For large spaces, LOS with ToF shows good promise.

In [52], the authors compared indoor localization using RFID, unmanned aerial vehicle (UAV), and 5G. For 5G, they used a carrier of $60 \mathrm{GHz}$ and a bandwidth of $2 \mathrm{GHz}$ and compared three conditions: LOS, obstructed LOS and obstructed LOS using beamforming. The results showed a localization error of only $2 \mathrm{~cm}$ for the LOS and $3 \mathrm{~cm}$ for the obstructed LOS. Beamforming made it more robust with only one anchor required. This problem was further studied in [57], where multi-path components in a 5G signal were used to localize indoor objects for assisted living.

3) Mapping: Mapping is useful in many applications, such as autonomous driving and geographical survey. Traditional methods use X-ray or radar [20]. They require dedicated hardware with high frequency, large bandwidth and antenna arrays. Consequently, they are extremely expensive and usually bulky. 5G signals can be used to capture the positions and shapes of objects in the environment for mapping at low cost but with sufficient accuracy, due to their mmWave carrier frequency and bandwidths of hundreds of $\mathrm{MHz}$.

In [75], an indoor mapping method was proposed using 5G new radio signals at $28 \mathrm{GHz}$. Phased arrays at both transmitter and receiver were steered towards different directions and the reflections from objects were used to reconstruct the environment. The processing is similar to that in Section III.A, where the periodogram was calculated and smoothed using a Gaussian kernel to map a corridor with 2 meters width and 60 meters length. The measurements were taken and processed by using a vector signal transceiver PXIe-5840 as RF transmitter and receiver at $3.5 \mathrm{GHz}$ and two signal generators N5183B-MXG with external mixers to up and down convert to $28 \mathrm{GHz}$ for OFDM signals with bandwidth 400 $\mathrm{MHz}$ and sub-carrier $120 \mathrm{kHz}$. The results showed impressive mapping performance.

In [76], joint localization and mapping was studied, where
5G signals were used in a message-passing method to jointly estimate the position, orientation of the UE, as well as the locations of any scatters or reflectors. With no prior knowledge assumed, the estimator was able to provide accurate singlesnapshot simultaneous localization and mapping even without LOS. In particular, the root mean squared error of the proposed method was much lower than the least squares method proposed in [77] and [78].

In [79], environment mapping was also studied for a $2 \mathrm{D}$ surface and a $3 \mathrm{D}$ space where $5 \mathrm{G}$ signals at $60 \mathrm{GHz}$ was transmitted as illuminator in a bistatic mode to perform mapping at the receiver. They used Analog Devices HMC6350 evaluation kit with HMC6300 board as transmitter and HMC6301 as receiver. Tektronix TSM4102A signal generator and DPO70404C oscillator were used to generate and record signals. The performance depends on the number of antennas, measurement noise, sparsity of signals etc. In [58], mapping for a 3D space was studied using reflected $5 \mathrm{G}$ OFDM system at $2 \mathrm{GHz}$ with a 64-element array at both transmitter and receiver. Similar to other works in this subsection, no changes to the existing $5 \mathrm{G}$ system were required for the mapping. They first proposed a FFT-based ranging method similar to frequency modulated continuous wave radar. Then ranging data from different channels were stitched to improve ranging resolution by increasing bandwidth. To verify this, they built a $5 \mathrm{G}$ prototype base station. Using a channel bandwidth of $100 \mathrm{MHz}, 8192$ sub-carriers with spacing 12.2 $\mathrm{kHz}$ to achieve 3D images of indoor scenes with angular resolution of 2 degrees and range resolution of $15 \mathrm{~cm}$, without affecting $5 \mathrm{G}$ communications.

In summary, the applications in this subsection are related to positioning and localization. These applications are related to each other, for example, outdoor environment mapping could be used for vehicle positioning while indoor environment mapping could be for UE positioning. Table VI summarizes application of $5 \mathrm{G}$ radio sensing in localization and positioning. The lessons learned can be summarized as follows. These works overwhelmingly use the RRSS measurements for high accuracy. However, further improvements could be achieved by using higher frequency or larger bandwidth, especially in [79] and [58]. Also, the stitching method from [58] suggests that, instead of using omni-directional antenna to cover the whole area, one may consider the use of directional antenna or beamforming to focus on different parts of the area and then stitch them together for better accuracy. Finally, very few of these works use machine learning or deep learning but these methods have been proved effective in other applications. Thus, they may be considered in localization and positioning.

\section{Other Applications}

Identification or detection of materials is an exciting use of $5 \mathrm{G}$ radio sensing. It has wide applications, such as concealed weapon detection or expired milk warning. Existing methods overwhelmingly use X-rays, radar, computed tomography (CT) or magnetic resonance imaging (MRI), which adopt extremely high frequency and large bandwidth signals for sensing. Hence, they are often expensive and require specialist 
TABLE VI

LOCALIZATION AND POSITIONING APPLICATIONS OF 5G RADIO SENSING

\begin{tabular}{|c|c|c|c|c|c|c|}
\hline Ref. & Application & Detectors & Frequency & Bandwidth & Methods & Accuracy \\
\hline$[15]$ & $5 \mathrm{G}$ radar & range, velocity & all $5 \mathrm{G}$ frequencies & all 5G bandwidths & correlator & varies \\
\hline$[51]$ & vehicular tracking & location & mmWave & unknown & $\begin{array}{l}\text { Bayesian } \\
\text { tracking filter }\end{array}$ & $\begin{array}{l}0.5 \text { meters for loca- } \\
\text { tion, } 0.02 \text { radians for } \\
\text { heading, } 0.3 \text { meters } \\
\text { for clock bias }\end{array}$ \\
\hline$[54],[60]$ & mapping & range, velocity & $28 \mathrm{GHz}$ & $400 \mathrm{MHz}$ & $\begin{array}{l}\text { periodogram, } \\
\text { self-interference } \\
\text { cancellation }\end{array}$ & unknown \\
\hline$[53]$ & UE positioning & location & $3.5 \mathrm{GHz}$ & $200 \mathrm{MHz}$ & $\begin{array}{l}\text { Extended } \\
\text { Kalman, } \\
\text { beamforming }\end{array}$ & $\begin{array}{l}0.1 \text { degrees error in } \\
\text { azimuth angle, } 3 \text { de- } \\
\text { grees error in eleva- } \\
\text { tion angle }\end{array}$ \\
\hline$[55]$ & UE positioning & location & $60 \mathrm{GHz}$ & $2.16 \mathrm{GHz}$ & $\begin{array}{l}\text { RSS-, ToF-, } \\
\text { AoA-trilateration }\end{array}$ & varies with parameters \\
\hline$[52],[57]$ & assisted living & location & $60 \mathrm{GHz}$ & $2 \mathrm{GHz}$ & $\begin{array}{l}\text { ML, tracking fil- } \\
\text { ter }\end{array}$ & $\begin{array}{l}0.02 \text { meters for LOS, } \\
0.03 \text { meters for NLOS }\end{array}$ \\
\hline$[75]$ & indoor mapping & location & $28 \mathrm{GHz}$ & $400 \mathrm{MHz}$ & $\begin{array}{l}\text { periodogram and } \\
\text { Gaussian kernel }\end{array}$ & unknown \\
\hline [76] & mapping & position, orientation & mmWave & unknown & $\begin{array}{l}\text { message passing } \\
\text { estimator }\end{array}$ & $\begin{array}{l}\text { error of } 1.8 \text { degrees in } \\
\text { orientation, } 7 \text { meters } \\
\text { in position }\end{array}$ \\
\hline [79] & imaging & region & $60 \mathrm{GHz}$ & $900 \mathrm{kHz}$ & scalar potential & varies with parameters \\
\hline$[58]$ & imaging & region & $28 \mathrm{GHz}$ & $100 \mathrm{MHz}$ & $\begin{array}{l}\text { radar processing } \\
\text { and stitching }\end{array}$ & $\begin{array}{lr}\text { angular resolution } 2 \\
\text { degrees and range } \\
\begin{array}{l}\text { resolution } \\
\text { meters }\end{array} & 0.15 \\
& \end{array}$ \\
\hline
\end{tabular}

training for operation. The use of $5 \mathrm{G}$ signals opens another horizon, as objects of different sizes, shapes and materials cause different reflection or diffraction of the $5 \mathrm{G}$ signals. In [73], liquid detection was studied using $5 \mathrm{G} \mathrm{C}$-band at $4.8 \mathrm{GHz}$. First, they used CSI to distinguish between suspicious and nonsuspicious liquids. Then, they identified the type of liquid. Finally, they distinguish different concentrations of alcohol. The transmitter and receiver were 1 meter away using signal generator and spectrum analyzer. The bandwidth was 100 $\mathrm{MHz}$ and transmission power was $-5 \mathrm{dBm}$. KNN was used to achieve an accuracy of $98 \%$ to detect suspicious liquids, $97 \%$ to identify the type and $94 \%$ to recognize the concentration of alcohol. In [74], a liquid-sensing ring resonator was designed at $36 \mathrm{GHz}$. It measured and analyzed the dielectric constant of the liquid to identify the liquid.

Humidity detection is another application of $5 \mathrm{G}$ radio sensing. In [82] and [83], on-body humidity sensing antenna was designed at the $38 \mathrm{GHz}$ frequency by detecting the dielectric constant of polyimide and changes in resonant frequency. UAV detection becomes a popular area in recent years, due to many unauthorized UAV flights and safety concerns. In [84], a 5G-based phased-array was designed for passive detection of UAVs. It used radar waveforms to detect a DJI Phantom 3 UAV with a range of more than 250 meters and SNR larger than $10 \mathrm{~dB}$ and an angular resolution of 8 degrees.

In summary, the high carrier frequency and the large channel bandwidth have equipped $5 \mathrm{G}$ signals with many benefits. In fact, the high frequency and large bandwidth of 5G have made 5G signals similar to conventional custom-made highresolution radar sensing signals. Thus, the $5 \mathrm{G}$ signals can be used to either detect and recognize large objects at fine resolution or small objects that are otherwise not possible using other wireless signals. The advantages and disadvantages of the works discussed above are self-evident from Tables IV - VI. For example, the approaches in [52], [57] require large bandwidth and high frequency to achieve high localization accuracy but the approach in [51] has low accuracy but less requirements on frequency and bandwidth. Thus, these advantages and disadvantages are not repeated here.

\section{Open Challenges}

To improve the accuracy of existing $5 \mathrm{G}$ radio sensing methods and to widen the use of $5 \mathrm{G}$ radio sensing in various applications, further investigation is required. Some open challenges in $5 \mathrm{G}$ radio sensing are discussed as follows. These challenges stem from the limitations of existing works that have been discussed above.

1) Outdoor Sensing: Most of the existing $5 \mathrm{G}$ radio sensing methods discussed above aim at indoor scenarios, except vehicle positioning. Indoor environments are more controllable with less uncertainty and higher probability of LOS in many cases. However, 5G provides coverage not only for indoors but also for outdoors. Thus, outdoor $5 \mathrm{G}$ radio sensing also requires attention, such as construction site safety and traffic monitoring. This is a challenge because outdoor environments are more complicated and more diversified and therefore, less controllable due to more scatters and higher dynamics. Intelligent reflecting surfaces could offer a solution for small areas by customizing the propagation environment [85]. 
2) Range: Related to the first challenge, most of the existing $5 \mathrm{G}$ sensing methods discussed above are only developed for uses in short range, when transmitter and receiver are separated a few meters away or at most tens of meters apart. This may be enough for indoor sensing. However, for outdoor applications and for large indoor venues, the sensing range needs to be increased, as the signal strength decays quickly with the distance. This could be achieved by increasing the transmission power but as the distance increases, it is likely to have more dynamics and the increased transmission power will increase interference too. Thus, $5 \mathrm{G}$ radio sensing will become more complicated for long range. This may require deep learning and data mining to extract as much information from the measurements as possible. Also, network densification in $5 \mathrm{G}$ alleviates this problem by increasing the density of access nodes.

3) Multiple Objects: Most of the existing 5G sensing works sense a single object. This is relatively easy, as the received signal is only affected by one object. However, there are scenarios where multiple objects need to be sensed in the same area, such as [65], [63] and [66]. Sensing multiple objects in the same area is relatively difficult, as their information is mixed in the received signal, but is necessary for some applications. References [65], [63] and [66] provided very elegant solutions by taking advantage of beam-steering to scan the space. However, this applies to multiple objects in a homogeneous environment. If they are in a heterogeneous environment, such as different persons staying in different rooms with different materials, $5 \mathrm{G}$ radio sensing will be difficult, as the propagation information for different persons may have different strengths so that one is stronger than the other in the same received signal. In this case, a scheme similar to successive interference cancellation may be useful.

4) Theoretical Models: Most existing 5G sensing works have studied the effects of different sensing parameters on the sensing accuracy. However, a systematic study of these effects is missing, as parameters could be correlated with others but these relationships are unknown. Also, most of these studies are heuristic via computer simulation. There is no theoretical model describing the explicit relationship between the sensing performance and sensing parameters. The lack of such model makes most $5 \mathrm{G}$ radio sensing designs ad hoc using trial-anderror. This issue is similar for WiFi sensing. However, since 5G uses the mmWave band, there are less multi-path components and hence, a more in-depth analysis may be possible by adopting the most recent advances in electromagnetic theories.

5) Joint Sensing and Communications: Most existing works consider $5 \mathrm{G}$ radio sensing only. However, in some applications, both sensing and communications functions may be required. It will be useful to develop joint sensingcommunications to improve efficiency. Sensing can use either ambient 5G signals dedicated to communications or 5G signals custom-made for sensing. The use of ambient 5G signals dedicated to communications makes sensing sub-optimal, as the optimal sensing waveform is different from the optimal communications waveform. The use of the optimal sensing waveform will require changes to existing $5 \mathrm{G}$ communications. Hence, a joint dual-functional or time-duplexed wave- form design for $5 \mathrm{G}$ radio sensing and $5 \mathrm{G}$ communications may be useful to reap the benefits of both radio sensing and communications.

6) Friendly to Sensing: Current $5 \mathrm{G}$ systems are designed for communications, not for radio sensing. Consequently, it is difficult for the sensing services to extract the RRSS, CSI or RSS measurements from the off-the-shelf $5 \mathrm{G}$ devices, as users are not expected to have access to these measurements. Currently, specialized hardware is required to retrieve these measurements. Thus, future $5 \mathrm{G}$ systems can be made more friendly to radio sensing by providing these measurements at the top layer for any users that require sensing services. This problem could be addressed in beyond $5 \mathrm{G}$ systems by upgrading the protocols.

7) Lack of Sensing Applications: Although many important applications of $5 \mathrm{G}$ radio sensing have been developed, as discussed before, there are still more to develop. For example, there has been no work on crowd counting using $5 \mathrm{G}$ signals. There has been no emotion recognition using $5 \mathrm{G}$ radio sensing. This is not surprising, as the $5 \mathrm{G}$ system has only been deployed recently, while WiFi and other generations of cellular signals have been in existence for a long time and therefore has more mature hardware and software. Nevertheless, given the many advantages that $5 \mathrm{G}$ signals have, it is expected that this area will attract more attention to develop more sensing applications. The quick roll-out of $5 \mathrm{G}$ systems and publications in this area, including this paper, may help tackle this.

8) Beamforming: Beamforming has played an important role in several $5 \mathrm{G}$ radio sensing works, such as [63], [65], [66] and [75], and may continue to play such a role in the future. $5 \mathrm{G}$ radio sensing uses the $5 \mathrm{G}$ signals that propagate through a changing environment to detect and classify such changes. Beamforming achieves additional spatial gain at the sensor by steering the beam pattern of the $5 \mathrm{G}$ signals to different directions for high-resolution sensing. It can be implemented by either reconfiguring the radiation pattern of an array via phase and amplitude adaptation or by physically steering the array to scan the space. Although its efficiency has been proven in [63], [65], [66] and [75], many 5G sensing works have not considered it, as beamforming is very energy-consuming and could incur high running cost. As the hardware cost reduces in the future, this problem will alleviate. Also, applications requiring high security and safety, such as collision avoidance and workplace safety, may adopt beamforming first.

9) Beyond 5G Sensing: The research on beyond $5 \mathrm{G}$ or $6 \mathrm{G}$ systems has recently started [86]. Although the key technologies for $6 \mathrm{G}$ are still under investigation, it is widely agreed that intelligent surfaces, artificial intelligence, mobile edge computing [87], fog computing [88], larger bandwidth and higher carrier frequency up to terahertz could be adopted. Thus, the open challenges of $5 \mathrm{G}$ radio sensing discussed above could be partly addressed in the evolution from $5 \mathrm{G}$ to $6 \mathrm{G}$. For example, the use of terahertz signals in $6 \mathrm{G}$ will make radio sensing even more accurate by having higher ranging resolution. The use of artificial intelligence will improve outdoor radio sensing. The use of mobile edge computing or fog computing will widen and ease the deployment of radio sensing services at 
TABLE VII

OPEN CHALLENGES AND POSSIBLE SOLUTIONS

\begin{tabular}{|c|c|}
\hline Challenges & Solutions \\
\hline outdoor sensing & intelligent reflecting surfaces \\
\hline range & deep learning, network densification \\
\hline multiple objects & advanced electromagnetic theories \\
\hline theoretical models & dual-functional designs \\
\hline joint sensing and communications & protocol upgrades \\
\hline friendly to sensing & roll-out of 5G \\
\hline lack of sensing applications & reduced hardware cost \\
\hline beamforming & combined radio sensing and 6G \\
\hline beyond 5G sensing & \\
\hline
\end{tabular}

the edge to relieve computational load and reduce latency. For these reasons, radio sensing will become even more important in 6G systems due to the increased demand for "smartness" in future networks and the increased accuracy of $6 \mathrm{G}$ radio sensing albeit more localized due to their larger bandwidth and higher frequency.

6G also offers a great opportunity for radio sensing by making the cellular systems more friendliness to sensing with easier access to measurements in the protocols and the development of joint sensing and communications to have higher integration of sensing and communications. Future 6G standards should include radio sensing to diversity its killer applications and to increase its efficiency. The main application scenario of radio sensing in $6 \mathrm{G}$ is localization and sensing, as outlined in the $6 \mathrm{G}$ white paper [89]. Other applications scenarios of radio sensing include joint sensingcommunications and autonomous driving. Table VII gives a list of the challenges and possible solutions discussed above.

\section{CONCLUSION}

This paper has provided an overview of most works on $5 \mathrm{G}$ radio sensing completed so far. Different sensing works have been discussed based on their applications in healthcare, safety, localization and others. This covers most works in the literature but is no complete list for all possible areas $5 \mathrm{G}$ radio sensing can help in the future. Hence, open challenges in $5 \mathrm{G}$ radio sensing have been discussed at the end. Moreover, future 6G systems will use even higher frequency and larger bandwidth to provide global wireless connection. These features will improve radio sensing further. Since the aim of this work is to provide an overview of existing works, no new simulation or experimental results have been provided and interested readers can refer to the references listed for such results.

\section{REFERENCES}

[1] X. Chen, J. Xiong, L. Chen, et al. "Sensing our world using wireless signals," IEEE Internet Computing, vol. 23, pp. 38 - 45, May-June 2019.
[2] E. Cianca, M. De Sanctis, and S. Di Domenico, "Radios as sensors," IEEE Internet of Things Journal, vol. 4, pp. 363 - 373, Apr. 2017.

[3] M. Beigl, A. Krohn, T. Zimmer, and C. Decker, "Typical sensors needed in ubiquitous and pervasive computing" Proc. First Int. Workshop Networked Sensing Systems (INSS), vol. 4, pp. 153-158, June 2004.

[4] S. Savazzi, S. Sigg, M. Nicoli, et al. "Device-free radio vision for assisted living: leveraging wireless channel quality information for human sensing," IEEE Signal Processing Mag., vol. 33, pp. 45 - 58, Mar. 2016.

[5] S. Savazzi, S. Sigg, F. Vicentini, et al. "On the use of stray wireless signals for sensing: a look beyond $5 \mathrm{G}$ for the next generation of industry," Computer, vol. 52 pp. 25 - 36, July 2019.

[6] J. Liu, H. Liu, Y. Chen, et al. "Wireless sensing for human activity: a survey," IEEE Commun. Surveys \& Tutorials, vol. 22, pp. 1629 - 1645, Third Quarter 2020

[7] W.Z. Khan, Y. Xiang, M.Y. Aalsalem, Q. Arshad, "Mobile phone sensing systems: a survey," IEEE Commun. Surveys \& Tutorials, vol. 15, pp. 402 - 427, First Q. 2013.

[8] S. Savazzi, S. Kianoush, V. Rampa, U. Spagnolini, "Cellular data analytics for detection and discrimination of body movements," IEEE Access, vol. 6, pp. 51484 - 51499, Sept. 2018.

[9] A. Gupta, R.K. Jha, "A survey of 5G network: architecture and emerging technologies," IEEE Access, vol. 3, pp. 1206 - 1232, July 2015.

[10] M. Simsek, A. Aijaz, M. Dohler, J. Sachs, "5G-enabled tactile internet," IEEE J. Select. Areas in Commun., vol. 34, pp. 460 - 473, Mar. 2016.

[11] S. Li, L. Xu, S. Zhao, "5G Internet of Things: a survey," Journal of Industrial Information Integration, vol. 10, pp. 1-9, June 2018.

[12] E. Dahlman, S. Parkvall, and J. Skld, 5G NR: The Next Generation Wireless Access Technology. Academic Press, Oxford, UK, 2018.

[13] 3GPP TS 38.104 v15.4.0, "NR: Base Station (BS) radio transmission and reception", Tech. Spec. Group Radio Access Network, Rel. 15, Dec. 2018.

[14] J.A. del Peral-Rosado, J.A. Lopez-Salcedo, S. Kim, et al. "Feasibility study of 5G-based localization for assisted driving," Proc. 2016 Int. Conf. on Localization and GNSS, Barcelona, Spain, June 28-30 2016.

[15] M. Kiviranta, I. Moilanen, J. Roivaninen, "5G radar: scenarios, numerology and simulations," Proc. 2019 Int. Conf. Military Commun. and Inform. Systems (ICMCIS), Budva, Montenegro, May 14-15, 2019.

[16] M. Zhu, J. Vieira, Y. Kuang, et al. "Tracking and positioning using phase information from estimated multi-path components," Proc. IEEE International Conference on Communication Workshops (ICCW), June 2015, pp. 712717.

[17] T.Q.S. Quek, M. Peng, O. Simeone and W. Yu, Cloud Radio Access Networks: Principles, Technologies and Applications, Cambridge University Press: Cambridge, UK, 2017.

[18] H. Jiang, C. Cai, X. Ma, et al. "Smart home based on WiFi sensing: a survey," IEEE Access, vol. 6, pp. 13317 - 13325, March 2018.

[19] D. Xu, B. Gaucher, U. Pfeiffer, J. Grzyb, Advanced Millimeter-Wave Technologies, Wiley: Chichester, UK. 2009. 
[20] B. Takacs, L.S. Sadovnik, V.A. Manasson, et al, "Real-time visualization using a 2D/3D imaging MMWave radar," Proc. SPIE 5297, Real-Time Imaging VIII, 18 May 2004.

[21] B.Kapilevich, M.Einat, A.Yahalom, et al. "Millemeter waves sensing behind walls - feasibility study with FEL radiation," Proc. FEL 2007, Novosibirsk, Russia, 2007.

[22] J.F. Federici, B. Schulkin, F. Huang, D. Gary, et al., "THz imaging and sensing for security applicationsexplosives, weapons and drugs," Semiconductor Science and Technology, vol. 20, 2005.

[23] H. Sarieddeen, M.-S. Alouini, T.Y.Al-Naffouri, "An overview of signal processing techniques for terahertz communications," arxiv.org/abs/2005.13176

[24] S. Dang, O. Amin, B. Shihada, M.-S. Alouini, "What should 6G be?", Nat Electron, vol. 3, pp. $20-29,2020$

[25] H. Sarieddeen, N. Saeed, T.Y. Al-Naffouri, M.-S. Alouini, "Next generation terahertz communications: a rendezvous of sensing, imaging, and localization,” IEEE Commun. Mag., vol. 58, pp. 69-75, May 2020.

[26] B. Paul, A.R. Chiriyath, D.W. Bliss, "Survey of RF communications and sensing convergence research," IEEE Access, vol. 5, pp. 252 - 270, Feb. 2017.

[27] S. Mazahir1, S. Ahmed, and M.-S. Alouini, "A survey on joint communication-radar systems," Frontiers in Communications and Networks, 2020.

[28] N. Cao, Y. Chen, X. Gu, W. Feng, "Joint bi-static radar and communications designs for intelligent transportation," IEEE Trans. Vehicular Technology, vol. 69, pp. 13060 - 13071, Nov. 2020.

[29] N. Cao, Y. Chen, X. Gu, W. Feng, "Joint radar-communication waveform designs using signals from multiplexed users," IEEE Trans. Commun., vol. 68, pp. 5216 - 5227, Aug. 2020.

[30] Y. Chen, X. Gu, "Time allocation for integrated bi-static radar and communication systems," IEEE Commun. Lett., Nov. 2020.

[31] F. Liu, C. Masouros, A. Li, T. Ratnarajah, J. Zhou, "MIMO radar and cellular coexistence: a power-efficient approach enabled by interference exploitation," IEEE Trans. Signal Processing, vol. 66, pp. 3681 - 3695, July 2018.

[32] E. Grossi, M. Lops, L. Venturino, "Joint design of surveillance radar and MIMO communication in cluttered environments," IEEE Trans. Signal Processing, Feb. 2020.

[33] Z.-M. Jiang, P.-C. Zhang, L. Huang, X. He, J.-H. Zhang and M. Rihan, "Transmit beampattarn optimization for automotive MIMO radar coexisted with communication in V2V networks," Sensors, vol. 20, 2020.

[34] S. Sodagari, A. Khawar, T.C. Clancy, R. McGwier, "A project based approach for radar and telecommunication systems coexistence," Proc. Globecom 2012, pp. 1-5, Anaheim, USA, Dec. 2012.

[35] A. Hassanien, M.G. Amin, Y.D. Zhang, and F. Ahmad, "Dual-function radar-communications: information embedding using sidelobe control and waveform diversity,"' IEEE Trans. Signal Processing, vol. 64, pp. 2168 - 2181, Apr. 2016

[36] F. Adib, H. Mao, Z. Kabelac, et al. "Smart homes that monitor breathing and heart rate," Proc. Conf. Hum. Factors Comput. Syst. (CHI), pp. 837846, 2015.

[37] Q. Pu, S. Gupta, S. Gollakota, and S. Patel, "Whole-home gesture recognition using wireless signals," Proc. Int. Conf. Mobile Comput. Netw. (Mobicom), pp. 27-38, 2013.

[38] T. Jayasri and M. Hemalatha, "Link quality estimation for adaptive data streaming in WSN," Int. J. Wireless Pers. Commn., vol. 94, no. 3, pp. 1543-1562, 2017

[39] M. Braun, "OFDM radar algorithms in mobile communication networks," Ph.D. dissertation, Karlsruhe Institute of Technology, 2014.

[40] S. Sigg, M. Scholz, S. Shi, et al. "RF-sensing of activities from noncooperative subjects in device-free recognition systems using ambient and local signals," IEEE Trans. Mobile Computing, vol. 13, pp. 907 921, Apr. 2014.

[41] B. Gholampooryazdi, I. Singh, S. Sigg, "5G ubiquitous sensing: passive environmental perception in cellular systems," Proc. 2017 IEEE 86th Vehicular Technology Conference (VTC-Fall), Toronto, Canada, Sept. 24 - 27, 2017.

[42] D. Haider, S.Y. Shah, O. Romain, et al. "Monitoring body motions related to Huntington disease by exploiting the 5G paradigm," Proc. UKChina Emerging Technologies Conf., Glasgow, UK, Aug. 21-22, 2019.

[43] S. Savazzi, R. Brondoliny, V. Rampa et al. "Motion discrimination by ambient cellular signals: machine learning and computing tools," Proc. 2019 IEEE 5th World Forum on Internet of Things, Limerick, Ireland, Apr. 15 - 18, 2019.

[44] X. Wang, J. Ellul, G. Azzopardi, "Elderly fall detection systems: a literature survey," Frontiers in Robotics and AI, pp. 1 - 23, June 2020.
[45] D. Haider, X. Yang, Q.H. Abbasi, "Post-surgical fall detection by exploiting the 5G C-Band technology for eHealth paradigm," Applied Soft Computing Journal, vol. 81, Aug. 2019.

[46] J.S. Khan, A. Tahir, J. Ahmad, et al. "5G-FOG: freezing of gait identification in multi-class softmax neural network exploiting $5 \mathrm{G}$ spectrum," Proc. Science and Information Conf., pp. 26 - 36, July 2020.

[47] B. Gholampooryazdi, S. Sigg, "Walking speed recognition from 5G prototype system," Proc. 2017 IEEE Int. Conf. Pervasive Computing and Commun. Workshops, Kona, USA, Mar. 13 - 17, 2017.

[48] B. Gholampooryazdi, Walking Speed Detection from 5G Prototype System, Aalto University, Finland.

[49] D. Haider, A. Ren, D. Fan, et al. "Utilizing 5G spectrum for healthcare to detect the tremors and breathing activity for multiple sclerosis," Trans. Emerging Telecommun. Tech., vol. 29, pp. 1 - 27, 2018.

[50] X. Yang, D. Fan, A. Ren, N. Zhao, M. Alam, "5G-based user-centric sensing at C-band," IEEE Trans. Industrial Informatics, vol. 15, pp. 30403047, May 2019.

[51] H. Kim, H. Wymeersch, N. Garcia, et al. "5G mmWave vehicular tracking," Proc. IEEE Asilomar Conf. on Signals, Systems, and Computers, Pacific Grove, USA, Oct. 28 - 31, 2018

[52] K. Witrisal, S. Hinteregger, J. Kulmer, E. Leitinger, P. Meissner, "Highaccuracy positioning for indoor applications: RFID, UWB, 5G, and beyond," Proc. 2016 IEEE Int. Conf. on RFID, Orlando, USA, May 3-5, 2016.

[53] M. Koivisto, A. Hakkarainen, M. Costa, P. Kela, K. Leppanen, M. Valkama, "High-efficiency device positioning and location-aware communications in dense $5 \mathrm{G}$ networks," IEEE Commun. Mag., vol. 55, pp. 188 - 195, Aug. 2017.

[54] C.B. Barneto, M. Turunen, S.D. Liyanaarachchi, et al. "High-accuracy radio sensing in $5 \mathrm{G}$ new radio networks: prospects and self-interference challenge," Proc. 2019 53rd Asilomar Conf. on Signals, Systems and Computers, Pacific Grove, USA, Nov. 3-6, 2019.

[55] F. Lemic, J. Martin. C. Yarp, et al., "Localization as a feature of mmWave communication," Proc. Int. Wireless Commun. and Mobile Computing Conf. (IWCMC), Paphos, Cyprus, Sept. 5-9, 2016.

[56] G. Soatti, S. Savazzi, M. Nicoli, et al. "Distributed signal processing for dense 5G IoT platforms: networking, synchronization, interference detection and radio sensing," Ad Hoc Networks, vol. 89, pp. 9 - 21, June 2019.

[57] K. Witrisal, P. Meissner, R. Leitinger, et al. "High-accuracy localization for assisted living: 5G systems will turn multipath channels from foe to friend," IEEE Signal Processing Mag., vol. 33, pp. 59 - 70, Mar. 2016.

[58] J. Guan, A. Paidimarri, A. Valdes-Garcia, B. Sadhu, "3D imaging using mmWave 5G signals," Proc. 2020 IEEE Radio Frequency Integrated Circuits Symposium, Los Angeles, USA, Aug. 4-6, 2020.

[59] H. Wymeersch, G. Seco-Granados, G. Destino et al. "5G mm-Wave positioning for vehicular networks," IEEE Wireless Commun., vol. 24, pp. 80 - 86, Dec. 2017.

[60] C.B. Barneto, T. Riihonen, M. Turunen, et al. "Full-duplex OFDM radar with LTE and 5G NR waveforms: challenges, solutions, and measurements," IEEE Trans. Microwave Theory and Techniques, vol. 67, pp. 4042 - 4054, Oct. 2019.

[61] R.S. Thoma, C. Andrich, G. Del Galdo, et al, "Cooperative passive coherent location: a promising $5 \mathrm{G}$ service to support road safety," IEEE Commun. Mag., vol. 57, pp. 86 - 92, Sept. 2019.

[62] I. Singh, S. Sigg, "Smart city environmental perception from ambient cellular signals," in Algorithms and Architectures for Parallel Processing, Springer, 2017.

[63] S. Sigg, S. Palipana, S. Savazzi, S. Kianoush, "Capturing humanmachine interaction events from radio sensors in Industry 4.0 environments," Proc. Business Process Management Workshops, pp. 430 - 435, Springer, Cham. 2020

[64] V. Rampa, S. Savazzi, F. Malandrino, "Opportunistic sensing in beyond5G networks: the opportunities of transformative computing," in the $5 G$ Italy Book 2019: A Multiperspective View of 5G, pp. 462 - 475, CNIT, 2019.

[65] S. Palipana, N. Malm, S. Sigg, "Beamsteering for training-free counting of multiple humans performing distinct activities," Proc. 2020 IEEE Int. Conf. on Pervasive Computing and Communications (PerCom), Austin, USA, Mar. 23 - 27, 2020.

[66] S. Palipana, S. Sigg, "Extracting human context through receiver-end beamforming," IEEE Access, vol. 7, pp. 154535 - 154545, Oct. 2019.

[67] S. Chen, J. Hu, Y. Shi, et al. "Vehicle-to-everything (v2x) services supported by LTE-based systems and 5G," IEEE Commun. Standards. Mag., vol. 1, pp. 70 - 76, July 2017. 
[68] M. Schmidhammer, S. Sand, M. Soliman, F. de Ponte Muller, "5G signal design for road surveillance," Proc. 2017 14th Workshop on Positioning, Navigation and Communications (WPNC), Bremen, Germany, Oct. 25 26, 2017.

[69] S. Denis, R. Berkvens, M. Weyn, "A survey on detection, tracking and identification in radio frequency-based device-free localization," Sensors, vol. 19, 5329, 2019.

[70] K. David, A. Flach, "Car-2-X and Pedestrian safety," IEEE Vehicular Technology Mag., vol. 5, pp. 70 - 76, Mar. 2010

[71] A. Jahn, K. David, and S. Engel, "5G/LTE based protection of vulnerable road users: detection of crossing a curb," 2015 IEEE 82nd Vehicular Technology Conference (VTC2015-Fall), Boston, USA, Sept. 6 - 9, 2015.

[72] A. Jahn M. Morold, K. David, "5G based collision avoidance - benefit from unobtrusive activities," Proc. 2018 European Conf. on Networks and Communications (EuCNC), Ljubljana, Slovenia, June 18 - 21, 2018.

[73] J. Deng, W. Sun, L. Guan, N. Zhao, et al. "Noninvasive suspicious liquid detection using wireless signals," Sensors, vol. 19, 2019.

[74] S.A. Qureshi, Z.Z. Abidin, A.Y.I. Ashyap, et al. "Liquid-sensing metamaterial ring resonator in millimeter-wave band for $5 \mathrm{G}$ applications," Proc. 2020 IEEE Student Conf. on Research and Development, Johor, Malaysia, Sept. 27 - 28, 2020.

[75] C.B. Barneto, T. Riihonen, M. Turunen, et al. "Radio-based sensing and indoor mapping with millimeter-wave 5G NR signals," Proc. 2020 Int. Conf. on Localization and GNSS (ICL-GNSS), Tampere, Finland, June 2-4, 2020.

[76] R. Mendrzik, H. Wymeersch, G. Bauch, "Joint localization and mapping through millimeter wave MIMO in 5G systems," Proc. IEEE Global Telecommun. Conf. (GLOBECOM), Abu Dhabi, UAE, Dec. 9 - 13, 2018

[77] A. Shahmansoori, G.E. Garcia, G. Destino, et al. "5G position and orientation estimation through millimeter wave MIMO, Proc. 2015 IEEE Globecom Workshops (GCWkshps), pp. 16, Dec 2015.

[78] A. Shahmansoori, G.E. Garcia, G. Destino, et al. "Position and orientation estimation through millimeter-wave MIMO in 5G systems," IEEE Trans. Wireless Commun., vol. 17, pp. 1822 - 1835, Mar. 2018.

[79] H. Li, "Sensing by leveraging millimeter wave communication networks," Proc. IEEE Global Telecommunications Conf. (GLOBECOM), Waikoloa, USA, Dec. 9 - 13, 2019.

[80] J.R. Landis, G.G. Koch, "The measurement of observer agreement for categorical data," Biometrics, vol. 33, pp. 159174, 1977.

[81] M.T. Hagan, H.B. Demuth, M.H. Beale, and O. De Jesus, Neural Network Design. Pws Pub. Boston, 1996.

[82] M.M. Rahman, M.A. Nayeem, S. Nahid, S.R.B. Alvee, R.R. Hasan, M.A. Rahman, "On-body humidity sensing antenna with polyimide for BAN applications over 5G networks," Proc. 2020 IEEE Int. IOT, Electronics and Mechatronics Conf., Vancouver, Canada, Sept. 9 - 12, 2020.

[83] X. Lin, B.-C. Seet, F. Joseph, "Wearable humidity sensing antenna for BAN applications over 5G networks," Proc. IEEE 19th Wireless and Microwave Technol. Conf. (WAMICON), Sand Key, USA, Apr. 9 - 10, 2018.

[84] Y. Wang, T. Phelps, K. Kibaroglu, et al. "28 GHz 5G-based phasedarrays for UAV detection and automotive traffic-monitoring radars," Proc. IEEE/MTT-S Int. Microwave Symposium, Philadephia, USA, June $10-15,2018$

[85] M. Di Renzo, A. Zappone, M. Debbah et al., "Smart radio environments empowered by reconfigurable intelligent surfaces: how it works, state of research, and the road ahead," IEEE Journal on Selected Areas in Communications, vol. 38, pp. 2450 - 2525, Nov. 2020.

[86] W. Saad, M. Bennis, M. Chen, "A vision of 6G wireless systems: applications, trends, technologies, and open research problems," IEEE Network, vol. 34, pp. 134 - 142, May/June 2019.

[87] Z. Zhao, Z. Ding, T. Q. S. Quek and M. Peng, "Edge artificial intelligence in $6 \mathrm{G}$ systems: theory, key techniques, and applications," China Communications, vol. 17, pp. iii-iv, Aug. 2020.

[88] M. Peng, T. Q. S. Quek, G. Mao, Z. Ding and C. Wang, "Artificialintelligence-driven fog radio access networks: recent advances and future trends," IEEE Wireless Communications, vol. 27, pp. 12-13, April 2020.

[89] A. Bourdoux, A. Noll Barreto, B. van Liempd et al. "6G White Paper on Localization and Sensing," available at https://arxiv.org/abs/2006.01779. 